\title{
Clasificación de la salinidad del suelo mediante imágenes de satélite y las redes neuronales artificiales.
}

\section{Classification of soil salinity using satellite images and artificial neural networks}

\author{
Lic. Rolando Renee Badaracco Meza y Dr. Joel Rojas Acuña
}

Universidad Nacional Mayor de San Marcos, Ap. Postal 14-0149.Lima, Perú

DOI: https://doi.org/10.33017/RevECIPeru2013.0001/

\begin{abstract}
Resumen
Los procesos de cambio climático han llamado obligatoriamente la atención de la ciencia en las últimas décadas, situaciones como la desertificación y sequia son temas de gran importancia. De aquí se desprendió la necesidad de estudiar la salinidad de los suelos y el índice de vegetación de diferencia normalizada (NDVI) usando imágenes de los sensores ETM+ y TM, estas imágenes fueron procesados usando el software ENVI 4.5 y el lenguaje de programación IDL. Para este propósito se implementó una red neuronal artificial con arquitectura de retropropagación de error, que permita clasificar la salinidad en los suelos, usando como datos de entradas imágenes de NDVI, y datos de entrenamiento de conductividad eléctrica del suelo. Las áreas de estudio fueron la comisión de regantes Muy Finca y los distritos de Lambayeque y Mórrope que pertenecen a la región Lambayeque-Perú. Para la arquitectura de la red neuronal se utilizó 2 capas ocultas presentando un error de entrenamiento de 0.27 , estos resultados fueron evaluados usando la matriz de confusión, el índice de kappa y el índice de exactitud global, obteniéndose un índice de kappa de 0.92 y el índice de exactitud global fue de $94.74 \%$. Finalmente se obtuvo una imagen clasificada de la distribución espacial de la salinidad en los suelos, la clasificación tuvo como resultado 3196.26 has como suelos muy fuertemente salinos, 2818.53 has como suelos fuertemente salinos, 3918.96 has como suelos moderadamente salinos, 10296.63 has como suelos ligeramente salinos y 2349.36 has como suelos no salinos.
\end{abstract}

Descriptores: Red Neuronal, NDVI, TM y ETM+

\section{Abstract}

The change climate processes have called attention of science in recent decades; situations such as desertification and drought are issues of great importance. Where it was the interest to study the soil salinity and the Normalized Difference Vegetation Index (NDVI) from images of the ETM + and TM sensors, these images were processed using the software ENVI 4.5 and IDL programming language. For this purpose, we implemented an artificial neural network with error back propagation architecture, for classifying soil salinity, using as input data of images NDVI and data soil electrical conductivity for the training. The areas studies were the commission irrigation Muy Finca and the districts of Morrope and Lambayeque belonging to the region Lambayeque-Peru. For the neural network architecture using 2 hidden layers presenting a training error of 0.27 , these results were evaluated using the confusion matrix, Kappa index and overall accuracy rate, obtaining a kappa index of 0.92 and over all accuracy rate was $94.74 \%$. Finally we obtained a classified image of the spatial distribution of salinity in soils, classification has resulted 3196.26 as strongly saline soils, 2818.53 you as strongly saline soils, 3918.96 you as moderately saline soils, 10296.63 you as slightly saline soils and 2349.36 have as non-saline soils.

Keywords: Neural Network, NDVI, TM and ETM + 


\section{Introducción}

Los estudios de salinización en Lambayeque son escasos, por lo que no se cuenta con información necesaria y actualizada; sin embargo, en el Valle Chancay-Lambayeque se han realizado en distintos periodos evaluaciones de salinidad de suelos con resultados sorprendentes y desalentadores como se muestra en la Tabla 1, [1]. La conductividad eléctrica del suelo (CE) ha sido el parámetro más extendido y el más ampliamente utilizado en la estimación de la salinidad. La CE refleja la concentración de sales solubles en la disolución. Para la clasificación de la salinidad del suelo se utilizó la Tabla 2 en donde muestra los rangos de la CE en extracto de 1:1.

Tabla 1. Salinidad en el Valle ChancayLambayeque

\begin{tabular}{|l|l|}
\hline 1963 & 14,230 has \\
\hline 1968 & 23,662 has \\
\hline 1975 & 42,022 has \\
\hline 1980 & 31,822 has \\
\hline 1990 & 40,258 has \\
\hline 2000 & 45,000 has \\
\hline
\end{tabular}

Tabla 2. Rangos generales de las plantas tolerantes a la salinidad, [2]

\begin{tabular}{|c|c|}
\hline Calificación & CE [1:1] $\left(\mathrm{dS} . \mathrm{m}^{-1}\right)$ \\
\hline No salino & $0.01-0.45$ \\
\hline Ligeramente salino & $0.45-1.5$ \\
\hline Moderadamente salino & $1.51-2.9$ \\
\hline Fuertemente salino & $2.91-8.5$ \\
\hline Muy fuertemente salino & $>8.5$ \\
\hline
\end{tabular}

Redes Neuronales y Perceptrón multicapa

El término red neuronal se aplica a una familia de modelos relacionada de manera aproximada que se caracteriza por un gran espacio de parámetro y una estructura flexible y que proviene de los estudios sobre el funcionamiento del cerebro, [3]. En la Figura 1 se muestra la gráfica arquitectónica de un perceptrón multicapa con dos capas ocultas y una capa de salida. La red que se muestra aquí está totalmente conectada. Esto significa que una neurona en cualquier capa de la red tiene una dirección hacia delante, de izquierda a derecha, [4].

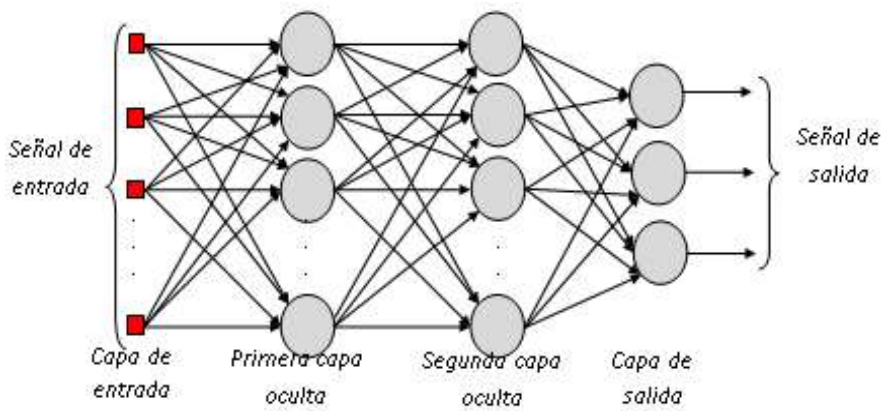

Figura 1. Arquitectura del perceptrón multicapa con dos capas ocultas

\section{El Algoritmo Back-Propagation}

Considerar un perceptrón multicapa con una capa de entrada, una o más capas ocultas, y una capa de salida que consiste en uno o más neuronas; el perceptrón multicapa es mostrado en la Figura 1. La señal error producido por la salida de la neurona $j$ se define por:

$$
e_{j}(n)=d_{j}(n)-y_{j}(n)
$$

Definimos al valor instantáneo de la energía de error para la neurona $j$ como

$$
\xi_{j}(n)=\frac{1}{2} e_{j}^{2}(n)
$$

Por lo tanto, el valor instantáneo de la energía de error total se obtiene al sumar $1 / 2 e_{j}^{2}(n)$ sobre todas las neuronas en la capa de salida; éstas son las únicas neuronas visibles para las cuales se pueden calcular directamente las señales de error. Escribimos entonces:

$$
\xi(n)=\frac{1}{2} \sum_{j \varepsilon C} e_{j}^{2}(n)
$$

Donde el conjunto $C$ incluye todas las neuronas en la capa de salida de la red. Sea $N$ el número total de patrones (ejemplos) contenidos en el set de entrenamiento. La energía de error cuadrático promedio se obtiene sumando $\xi(n)$ sobre cada $n$ y luego normalizando con respecto al tamaño $N$ del set, como se muestra en:

$$
\xi_{a v}(n)=\frac{1}{2} \sum_{n=1} \xi(n)
$$

La energía de error instantánea $\xi(n)$, y por lo tanto la energía de error promedio $\xi_{a v}$, es función de todos los parámetros libres (pesos de conexión y umbrales) de la red.

\section{Ubicación de las áreas de estudio}


Las áreas de estudio fueron la comisión de regantes Muy Finca y los distritos de Lambayeque y Mórrope que pertenecen a la región Lambayeque que está ubicada en la costa norte del Perú. En la Figura 2 se muestra la ubicación de las 3 áreas de estudio.

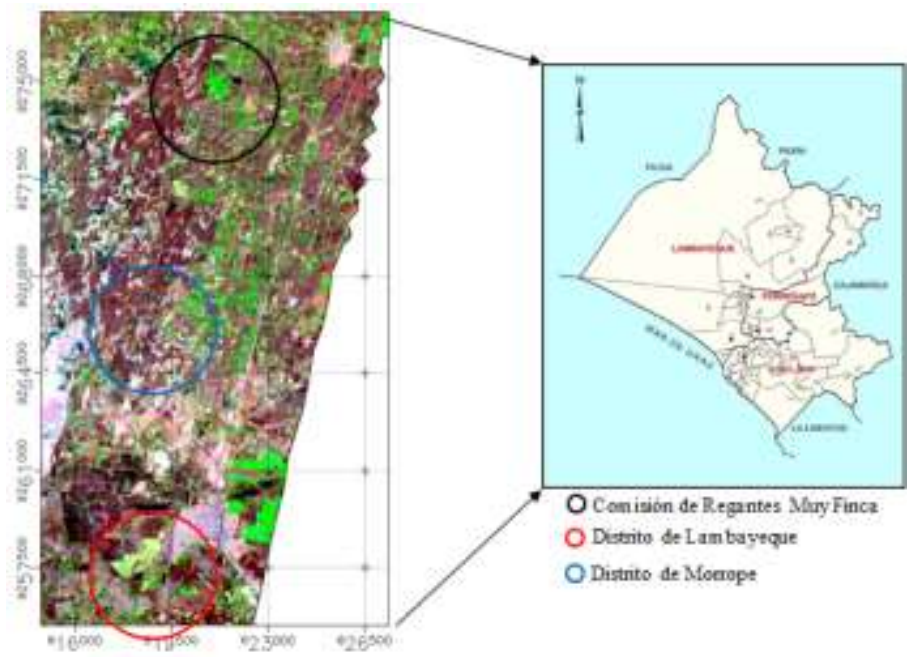

Figura 2. Ubicación de las áreas de estudio en la región de Lambayeque

Adquisición de las imágenes satelitales

Las imágenes utilizadas fueron de los satélites Landsat 5 y Landsat 7, estas imágenes fueron obtenidas gracias a la cortesía de la United States Geological Survey (USGS), en total se utilizaron 46 imágenes, 3 imágenes del satélite Landsat 5 y 43 imágenes del satélite Landsat 7, [5].

\section{Adquisición de muestras de campo}

En las áreas de estudio se ubicó puntos de muestreo debidamente georeferenciadas con la ayuda de un GPS. Se extrajo las muestras de suelo de $0.0-0.30 \mathrm{~m}$ de profundidad, que fueron trasladadas al laboratorio de suelos de la Facultad de Agronomía de la UNPRG, en donde se determinó la Conductividad Eléctrica de 190 muestras distribuidas como se muestra en la Tabla 3.

Tabla 3. Distribución de las muestras de campo en las áreas de estudio

\begin{tabular}{|c|c|c|}
\hline Área de estudio & $\begin{array}{c}\text { Fecha de } \\
\text { adquisición }\end{array}$ & $\begin{array}{c}\text { Total de } \\
\text { muestras }\end{array}$ \\
\hline $\begin{array}{c}\text { Comisión de } \\
\text { Regantes Muy Finca }\end{array}$ & $17 / 10 / 2007$ & 77 \\
\hline $\begin{array}{c}\text { Distrito de } \\
\text { Lambayeque }\end{array}$ & 36 \\
\hline Distrito de Morrope & $13 / 10 / 2007$ & 77 \\
\hline
\end{tabular}

\section{Metodología}

Calibración radiometrica de las imágenes TM y ETM+

Las imágenes obtenidas de los sensores TM y ETM+ contiene números digitales ( 0 a 255). Estos valores están relacionados directamente con la radiancia. La conversión de los números digitales (ND) de una imagen a radiancia (L) está dado por la siguiente ecuación [6].

$$
\begin{aligned}
& N D=\left(\frac{N D_{\text {max }}}{L_{\text {max }}-L_{\text {min }}}\right) x\left(L-L_{\text {min }}\right) \\
& L=\left(\frac{L_{\text {max }}-L_{\text {min }}}{N D_{\text {max }}}\right) x N D+L_{\text {min }}
\end{aligned}
$$

Dónde:

L: Radiancia (W/m².sr.um).

ND: Número digital para cada pixel de la imagen.

$L_{\max } L_{\min }$ : Constantes de Calibración.

$\mathrm{ND}_{\max }$ : Número digital máximo de la imagen.

\section{Corrección atmosférica usando el módulo FLAASH}

FLAASH (Fast Line-of-Sight Atmospheric Analysis of Spectral Hypercubes) toma como base una ecuación estándar para la radiancia espectral en un pixel en el sensor, $L$, aplicado para el rango de longitud de onda solar (la emisión térmica es despreciada), en materiales Lambertianas 0 equivalents, [7].

$$
L=\left(\frac{A \rho}{1-\rho_{e} s}\right)+\left(\frac{B \rho}{1-\rho_{e} s}\right)-L_{a}
$$

Dónde:

$\rho$ : es la reflectancia superficial del pixel.

$\rho_{e}$ : es la reflectancia superficial promedio del pixel y la región de entorno.

$s$ : es el albedo esférico de la atmósfera.

$L_{a}$ : es parte de la radiancia dispersada por la atmósfera.

$A$ y $B$ : son coeficientes que dependen de las condiciones atmosféricas y geométricas pero no depende de la superficie.

\section{Clasificación supervisada}


Para la clasificación supervisada se utilizó el software ENVI 4.5 y el lenguaje de programación IDL utilizando el algoritmo de back-propagation. En la Tabla 4 se detallan los parámetros usados para la clasificación supervisada de la salinidad de los suelos.

Tabla 4. Parámetros utilizados en la Red Multicapa

\begin{tabular}{|c|c|}
\hline \multicolumn{2}{|c|}{ Parámetros de la Red Multicapa } \\
\hline \# de Iteraciones & 10000 \\
\hline Tasa de aprendizaje & 0.01 \\
\hline Momento & 0.9 \\
\hline Función de activación & $\begin{array}{c}\text { Función } \\
\text { logística }\end{array}$ \\
\hline \# de neuronas en la capa de entrada & 46 \\
\hline \# de neuronas en la capa de salida & 5 \\
\hline \# de capas ocultas & 1 y 2 \\
\hline
\end{tabular}

\section{Resultados y discusión}

Error de entrenamiento de la red multicapa mediante el algoritmo de back-propagation

En la Figura 3 se muestra el error del entrenamiento de la red multicapa de 46 neuronas en la capa de entrada, 5 neuronas en la capa de salida y 2 capas ocultas. El error del entrenamiento después de 10000 iteraciones fue de 0.27.

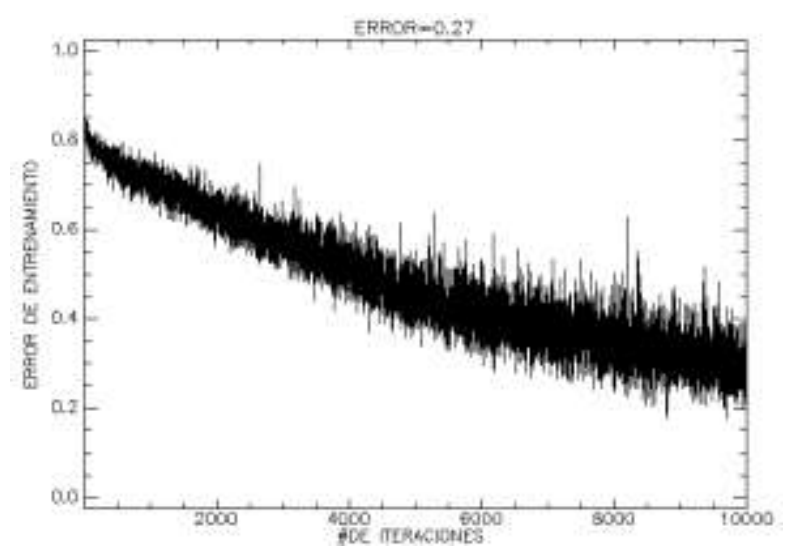

Figura 3. Error de entrenamiento con dos capas ocultas.

En la Tabla 5 se muestra la matriz de confusión, el índice de exactitud global y el índice de kappa. Las siguientes variables son usadas en la matriz de confusión: R\#1=Muy Fuertemente Salino, R\#2= Fuertemente Salino, R\#3=Moderadamente Salino, R\#4=Ligeramente Salino, R\#5=No Salino.

Tabla 5. Matriz de confusión para la red multicapa con dos capas ocultas

\begin{tabular}{|c|c|c|c|c|c|}
\hline Clases & $\mathrm{R} \# 1$ & $\mathrm{R} 2$ & $\mathrm{R}$ 3 & $\mathrm{R} 44$ & $\mathrm{R}$ 5 \\
\hline $\mathrm{R} \# 1$ & 18 & 0 & 0 & 0 & 1 \\
\hline $\mathrm{R} \# 2$ & 0 & 19 & 0 & 0 & 1 \\
\hline $\mathrm{R} 3$ & 0 & 0 & 18 & 0 & 1 \\
\hline $\mathrm{R} 44$ & 0 & 9 & 2 & 89 & 2 \\
\hline $\mathrm{R}$ 5 & 1 & 0 & 1 & 1 & 36 \\
\hline Total & 19 & 19 & 21 & 90 & 41 \\
\hline
\end{tabular}

El índice de exactitud global es igual a $(180 / 190)=$ $94.7368 \%$ y el índice de kappa es de 0.9238 .

En la Figura 4, se muestra la clasificación espacial de la salinidad del suelo. Las áreas de color rojo pertenecen a la clase de zonas muy fuertemente salinos, las áreas de color verde pertenecen a la clase de zonas fuertemente salinos, las áreas de color azul pertenecen a la clase de zonas moderadamente salinos, las áreas de color amarillo pertenecen a la clase de zonas ligeramente salinos y las áreas de color cyan pertenecen a la clase de zonas no salino. Hay un total de 35514 pixeles clasificados como suelos muy fuertemente salino equivalente a 3196.26 has. Hay un total de 31317 pixeles clasificados como suelos fuertemente salino equivalente a 2818.53 has. Hay un total de 43544 pixeles clasificados como suelos moderadamente salino equivalente a 3918.96 has. Hay un total de 114407 pixeles clasificados como suelos ligeramente salino equivalente a 10296.63 has. Hay un total de 26104 pixeles clasificados como suelos no salino equivalente a 2349.36 has.

\section{Conclusiones}

La presente metodología cumplió con los objetivos de clasificar la salinidad en los suelos con el uso de imágenes de satélite y los datos in situ de conductividad eléctrica. EI NDVI es un parámetro físico muy importante ya que está relacionado con la conductividad eléctrica del suelo. Se recomienda que para futuras investigaciones las muestras de campo deban ser tomadas en plena fase terminal o final de su desarrollo vegetativo (al $50 \%$ de la floración), etapas en que las plantas desarrollan la más intensa actividad fisiológica, poseen una mayor cobertura vegetativa y en consecuencia se logra un mayor reflejo de la luz solar. Ya que en esta época, el efecto de la sales del suelo en las raíces de las plantas y en toda la planta es mayor que en etapas fisiológicas previas, por lo que es más fácil discriminar las áreas afectadas. Para un mejor estudio se debe tomar áreas agrícolas más extensas en la región de Lambayeque y con un 

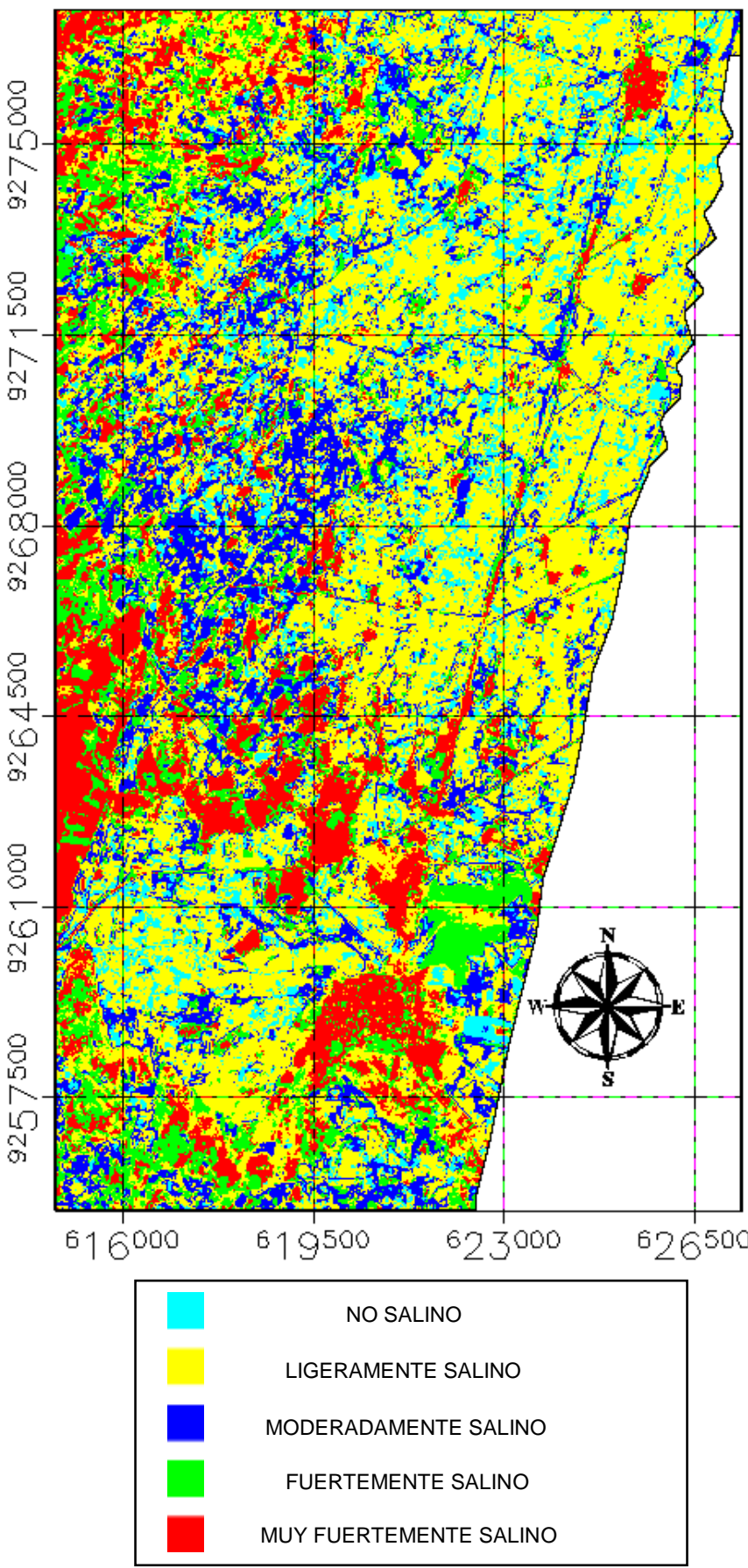

Figura 4. Clasificación supervisada de la salinidad en los suelos.

mayor número de muestras de campo repartidas por toda el área a investigar. Para futuras investigaciones en estas áreas y con el propósito de obtener resultados más precisos, se debe utilizar imágenes satelitales de sensores remotos con mayor resolución espacial.

\section{Agradecimientos}

Un agradecimiento especial al Proyecto Cátedra UNMSM-CONCYTEC: "Teledetección en
Desertificación y Sequía" (R.P.N³28-2011-P) por la subvención de una beca de tesis de maestría en Geofísica que recibió el autor RRBM durante el año 2012 y a la Asociación Familia Zúñiga y Rivero.

\section{Referencias}

[1] Gobierno regional de Lambayeque (2011). Plan de Desarrollo Regional Concertado Lambayeque. Pág. 285.

[2] Fuentes J. L. (1999). El suelo y los fertilizantes, 5a edición. Ed. Mundi-Prensa. Madrid Barcelona - México. Pág. 350.

[3] Bishop C. M. (1995). Neural Networks for Pattern Recognition, Oxford University Press, Walton Street, Oxford. Pág. 482.

[4] Haykin S. (1999). Neural Networks. A Comprehensive Foundation, Second Edition. Pretince Hall. Pág. 906.

[5] Chander G., Markham B. y Helder D. (2009). Summary of current radiometric calibration coefficients for Landsat MSS, TM, ETM+, and EO-1 ALI sensors, Remote sensing of environment 113. Pág. 893-903.

[6] Williams D., (2009). Landsat-7 Science Data User's Handbook, National Aeronautics and Space Administration. Pág. 186.

[7] Matthew, M. W., Adler-Golden S. M., Berk A., Richtsmeier S. C., Levine R. Y., Bernstein L.S., Acharya P. K., Anderson G. P., Felde G. W., Hoke M. P., Ratkowski A., Burke H. H., Kaiser R. D. y Miller D. P. (2000). Status of Atmospheric Correction Using a MODTRAN4-based Algorithm, SPIE Proceeding, Algorithms for Multispectral, Hyperspectral, and Ultraspectral Imagery VI, Vol. 4049, Pág. 199-207.

E-mail: badaracco meza@hotmail.com 